\title{
Adaptability index: quantifying CT tube current modulation performance from dose and quality informatics
}

\author{
F. Ria ${ }^{1,2,3}$, J.M. Wilson ${ }^{1}$, Y. Zhang ${ }^{1}$, E. Samei ${ }^{1}$
}

(1) Carl E. Ravin Advanced Imaging Labs and Clinical Imaging Physics Group, Duke University Heath System, Durham NC 27705; (2) Dipartimento Diagnostica per Immagini, Centro Diagnostico Italiano, Milan ITALY; (3) Alumnus progettoDiventerò di Fondazione Bracco, Milan ITALY

\begin{abstract}
The balance between risk and benefit in modern CT scanners is governed by the automatic adaptation mechanisms that adjust x-ray flux for accommodating patient size to achieve certain image noise values. The effectiveness of this adaptation is an important aspect of CT performance and should ideally be characterized in the context of real patient cases. Objective of this study was to characterize CT performance with an index that includes image-noise and radiationdose across a clinical patient population.

The study included 1526 examinations performed by three scanners, from two vendors, used for two clinical protocols (abdominopelvic and chest). The dose-patient size and noise-patient size dependencies were linearized, and a 3D-fit was performed for each protocol and each scanner with a planar function. In the fit residual plots the Root Mean Square Error (RMSE) values were estimated as a metric of CT adaptability across the patient population.

The RMSE values were between $0.0344 \mathrm{HU}^{1 / 2}$ and $0.0215 \mathrm{HU}^{1 / 2}$ : different scanners offer varying degrees of reproducibility of noise and dose across the population. This analysis could be performed with phantoms, but phantom data would only provide information concerning specific exposure parameters for a scan: instead, a general population comparison is a way to obtain new information related to the relevant clinical adaptability of scanner models.

A theoretical relationship between image noise, $\mathrm{CTDI}_{\mathrm{vol}}$ and patient size was determined based on real patient data. This relationship may provide a new index related to the scanners' adaptability concerning image quality and radiation dose across a patient population.
\end{abstract}

Key words: image noise, radiation dose, patient population, CT performance

Medical Imaging 2017: Physics of Medical Imaging, edited by Thomas G. Flohr,

Joseph Y. Lo, Taly Gilat Schmidt, Proc. of SPIE Vol. 10132, 101322N · C 2017 SPIE

CCC code: $1605-7422 / 17 / \$ 18 \cdot$ doi: $10.1117 / 12.2255631$

Proc. of SPIE Vol. 10132 101322N-1 


\section{INTRODUCTION}

CT diagnostic protocols should be designed and optimized to balance image quality and radiation dose and clinically acceptable image quality has become an issue as dose reduction strategies. The aim of optimization must be to keep radiation exposure "as low as reasonably achievable" (ALARA) while ensuring acceptable clinical images [1-2]. Therefore, behind every optimization action, there is need of a risk/benefit evaluation. The effort to contextually compare the image quality and radiation dose is a natural progression of the optimization principle [2] because the acquisition parameters are directly related to the clinical benefit: dose is correlated with patient risk.

The balance between risk and benefit in modern CT scanners is governed by the automatic adaptation mechanisms that adjust X-ray flux for accommodating patient size to achieve certain image noise values [3], [4], [5], [6]. The effectiveness of this adaptation is an important aspect of CT performance and should ideally be characterized in the context of real patient cases because a phantom study may only provide information related to the scanner performance in a highlyconstrained setup [7].

The objective of this study was to characterize CT tube current modulation performance with a new metric that includes image noise and radiation dose as a function of patient size. This methodology can be implemented within dose monitoring programs that collect patient diameter, dose and image noise values, which allows CT performance characterization across patient populations.

\section{METHODS}

The study included 1526 examinations (January 2015 and June 2015 through January 2016) performed by three CT scanners: GE Healthcare Discovery CT750HD, GE Healthcare Lightspeed VCT, and Siemens SOMATOM definition Flash used for two routine clinical protocols (abdominopelvic with contrast and chest without contrast). The three scanners were anonymized and they are identified as CT1, CT2 and CT3. All data presented in this work came from the Duke Health dose monitoring program (DHDMP) [8], [9].

The DHDMP stores patient width, thickness and effective diameter as well as dose and image noise values for all CT studies. After the CT images are acquired on the scanners, together with the structured dose report, they are sent to both PACS and the Dose Monitoring Server, where images are temporarily stored and then analyzed for patient sizes and noise. The scout images are contoured to calculate the patient sizes, and the tomographic images are used to calculate the noise. The tomographic images are first applied a threshold of $-100 \sim 300 \mathrm{HU}$ to isolate soft tissue (relatively uniform and artifact free compared to other tissue types) for noise calculation. For each slice, an ROI is identified around each pixel, generating a standard deviation and a histogram of standard deviation map across the slice. Noise of that slice is computed as the value corresponding to the peak of the histogram. The whole CT image set was binned to ten groups and the noise calculation process is conducted automatically for the first slice for each bin. The final noise value for the image set is computed as the mean of the noise values.

In order to consider only the diagnostic series, scout, iterative reconstruction, contrast monitoring, and contrast premonitoring series were excluded. Since the proposed method needs to be applied to consistent populations in term of scan parameters and convolution kernels, for each protocol, we selected the most frequent exams with the same $\mathrm{kVp}$, slice thickness, total collimation width, pitch, convolution kernel, and image quality indicator. GE Healthcare and Siemens Healthcare scanners use two distinct angular automatic tube current modulation system(ATCM) methodologies with target image quality indicators corresponding to varying degrees of image quality. The image quality indicators are noise index (NI) for GE Healthcare and image quality reference effective tube current-time product (Q) for Siemens Healthcare scanners. In this work, we included the corresponding level of image quality index for each protocol. A summary of examinations included in the study is reported in Table 1. 
Table 1. Summary of examinations included in the study sorted by scanners, clinical protocol, and scan parameters.

\begin{tabular}{cccccccc}
\hline \hline CT scanners & Protocol & $\begin{array}{c}\text { Number of } \\
\text { patients }\end{array}$ & $\begin{array}{c}\text { Slice } \\
\text { thickness } \\
(\mathbf{m m})\end{array}$ & $\begin{array}{c}\text { Total } \\
\text { collimation } \\
\text { width (mm) }\end{array}$ & kVp & Pitch & NI-Q \\
\hline \hline $\begin{array}{c}\text { GE Discovery } \\
\text { CT750HD }\end{array}$ & $\begin{array}{c}\text { A\&P with } \\
\text { contrast } \\
\text { Chest without } \\
\text { contrast } \\
\text { A\&P with } \\
\text { contrast }\end{array}$ & 266 & 0.625 & 40 & 120 & 1.375 & 220 \\
$\begin{array}{c}\text { GE Lightspeed } \\
\text { VCT }\end{array}$ & 414 & 5 & 40 & 120 & 1.375 & 192 \\
$\begin{array}{c}\text { Chest without } \\
\text { contrast } \\
\text { A\&P with } \\
\text { SIEMENS }\end{array}$ & 48 & 523 & 5 & 40 & 120 & 1.375 & 150 \\
$\begin{array}{c}\text { SOMATtrast } \\
\text { Definition Flash }\end{array}$ & $\begin{array}{c}\text { Chest without } \\
\text { contrast }\end{array}$ & 68 & 5 & 40 & 120 & 1.375 & 160 \\
\hline \hline
\end{tabular}

To evaluate the scanners' adaptability across a population, dose-patient size dependency and noise-patient size dependency were linearized. For this work, we chose to use the $\mathrm{CTDI}_{\mathrm{vol}}$ as a dose index as a highly scanner-independent metric. The radiation dose is directly related to the attenuating diameter $C T D I_{v o l} \propto e^{d}$ [8], where $d$ is the phantom diameter. Thus $\ln C T D I_{v o l}$ was used as a linear descriptor of CTDI ${ }_{\mathrm{vol}}$ as a function of diameter

In terms of noise, a preceding study [10] reported an empirical equation that relates noise $\sigma$, phantom diameter, and tube current $(m A)$ as

$$
\ln \sigma=\alpha_{0}+\alpha_{1} d+\alpha_{2} \ln (m A)+\alpha_{3} d^{2}+\alpha_{4}(\ln (m A))^{2}+\alpha_{5} d \ln (m A)
$$

where $\alpha_{1}, \alpha_{2}, \alpha_{3}, \alpha_{4}, \alpha_{5}$ are fitting parameters. Furthermore, an investigation of the ImPACT Group [11] showed a linear proportionality between the logarithm of $m A$ and phantom diameter as

$$
\ln (m A)=m d+q,
$$

where $m$ is the slope, and $q$ is the intercept of the curve. From equations (1) and (2), it follows that noise can be linearized as a function of diameter in terms of $\sqrt{\ln \sigma}$.

A 3D-fit of $\sqrt{\ln \sigma}$ across $\ln C T D I_{v o l}$ and patient diameter was performed for each protocol and each scanner with a planar $1^{\text {st }}$ degree function. This will characterize the overall dependency of noise versus CTDI $_{\mathrm{vol}}$ for each scanner across a population. We used the Curve Fitting Toolbox ${ }^{\mathrm{TM}}$ application in MATLAB ${ }^{\circledR}$ R2015b software [12]: the Root Mean Square Error (RMSE) values were estimated in the residuals' plots as a consistency metric of system adaptability across the study cases.

\section{RESULTS}

Figures 1, 2, and 3 show the analysis performed in this study for CT1 abdomen and pelvis with contrast protocol data. In particular, Figure 1 shows the 3D-fit plot of $\sqrt{\ln \sigma}$ across $\ln C T D I_{v o l}$ and patient diameter with a planar $1^{\text {st }}$ degree function. Figure 2 shows the same 3D-fit of dose versus patient diameter with isocontours of noise, and Figure 3 is the 
related fit residuals plot. The analysis was performed for all the six populations involved in this study and the RSME values are summarized in Table 2.

Table 2. Summary of RMSE values of the 3D-fits for each scanner and each protocol involved in this study.

\begin{tabular}{cc}
\hline \hline Code & Fit RMSE $\left(\mathbf{H U}^{\mathbf{1 / 2}}\right)$ \\
\hline \hline $\begin{array}{c}\text { CT1 abdominopelvic } \\
\text { with contrast }\end{array}$ & 0.0334 \\
CT1 chest without \\
$\begin{array}{c}\text { contrast } \\
\text { CT2 abdominopelvic } \\
\text { with contrast }\end{array}$ & 0.0344 \\
CT2 chest without \\
$\begin{array}{c}\text { contrast } \\
\text { CT3 abdominopelvic } \\
\text { with contrast }\end{array}$ & 0.0385 \\
CT3 chest without \\
contrast
\end{tabular}

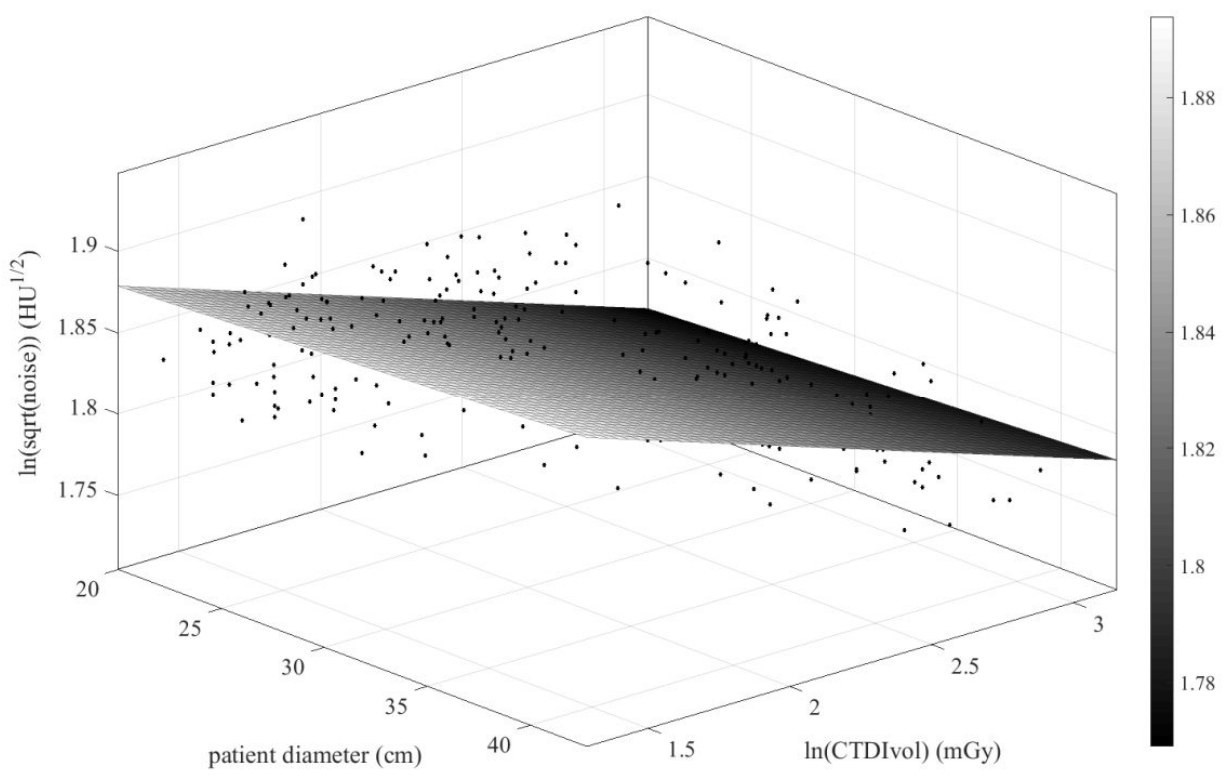

Figure 1. Plot of the 3D fit for CT1 abdominopelvic with contrast protocol. For each exam (black markers) the three axis reports patient diameter in $\mathrm{cm}$, dose information $\left(\ln C T D I_{v o l}\right)$ in $m G y$, and the noise value information in $\mathrm{HU}^{1 / 2}(\sqrt{\ln \sigma})$ 


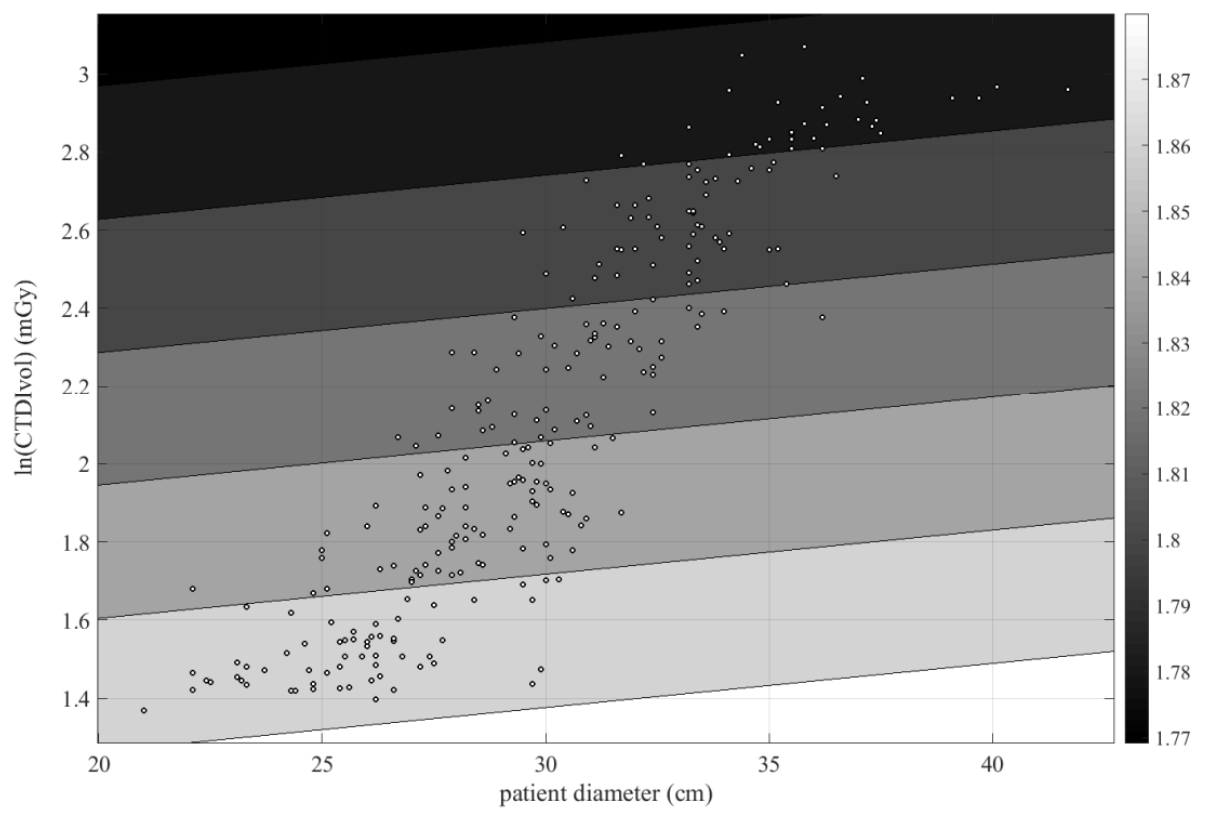

Figure 2. CT1 A\&P with contrast protocol 3D fit. The color bar represents the noise information in $\mathrm{HU}^{1 / 2}(\sqrt{\ln \sigma})$, the horizontal axis reports patient diameter in $\mathrm{cm}$, and vertical axis represents dose information (ln $\left.\mathrm{CTDI}_{\mathrm{vol}}\right)$ in $\mathrm{mGy}_{\text {. }}$

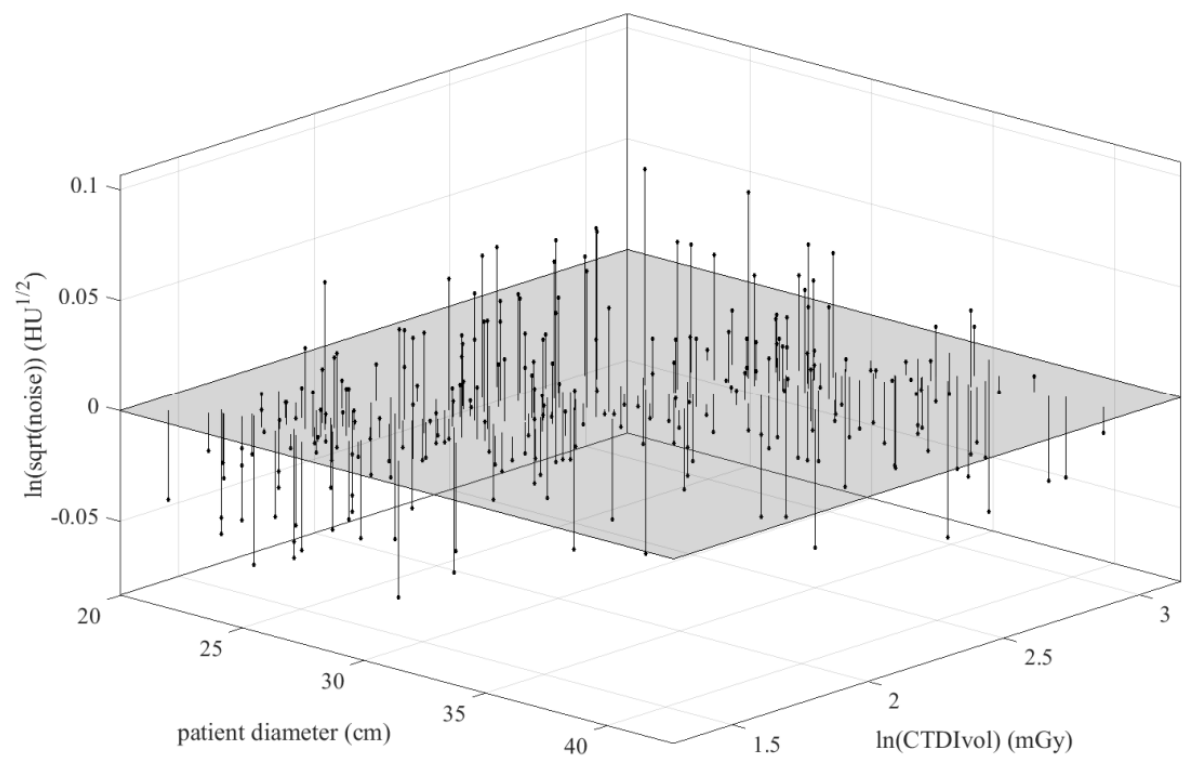

Figure 3. Plot of the 3D fit residuals for CT1 abdominopelvic with contrast protocol. The three axis reports patient diameter in $c m$, dose information $\left(\ln C T D I_{v o l}\right)$ in $m G y$, and the noise value information in $\mathrm{HU}^{1 / 2}(\sqrt{\ln \sigma})$ 


\section{DISCUSSION}

We performed an analysis to compare the performance of different CT scanners with a new metric that simultaneously incorporates image quality information (noise) and radiation dose information $\left(\mathrm{CTDI}_{\mathrm{vol}}\right)$ throughout a population of patients. Although this analysis could be performed with phantoms, phantom data would only provide information concerning specific exposure parameters for a scan [13]: instead, a general population comparison is a way to obtain new information related to the patient adaptability of different scanners. To our knowledge, such a comparison across manufacturers that includes dose, noise and patient size information has not been previously reported.

The data show different scanner dependencies in terms of adaptability: the RMSE values for the three scanners are between $0.0385 \mathrm{HU}^{1 / 2}$ and $0.0215 \mathrm{HU}^{1 / 2}$. Thus, different scanners offer different degrees of reproducibility of noise and dose values across the population and lower RSME values are associated with better performance reproducibility. In general, for increasing values of dose and patient effective diameter, noise values also increase, with the exception of chest protocol performed with CT1. The study is not affect by the different trends because we have performed the analysis in six populations with consistent scanner parameters and convolution kernels.

Larson et al. [7] introduced a mathematical CT radiation dose optimization model based on the minimization of the radiation dose that provides a constant image noise level for different patient sizes. Their model, while innovative and informative, did not consider that the inter-scanner performance and dose administered is not constant across a population. Different scanners have different levels of adaptability. A previous study [9] used the DHDMP algorithm to estimate the variability of noise, SSDE, and effective diameter data across the three scanner models involved in this study: the median effective diameter differed by $2-8 \%$, the median SSDE differed by $9-33 \%$, and the median noise values differed by $15-35 \%$, between the three scanner models. In this work, we have shown that the variability cannot be interpreted only in terms of absolute values because scanner performance varies when radiation dose is modulated to achieve consistent image quality across a population.

This work is limited in multiple aspects. The work shown here includes only three scanners by two manufacturers and only two different clinical protocols for specific $\mathrm{kVp}$, slice thickness, total collimation width, pitch, image quality indicator and convolution kernel. But, the method described can be implemented to investigate the 3D relationship between noise, radiation dose, and patient size, even for different scan parameters and different image quality indicator values. It should be noted that the results do not show absolute values of image quality and radiation dose across a population but only the difference between different vendors in terms of patient variability. Also, the study does not consider the maximum and minimum tube current values that are set up in certain CT devices. Despite the radiographers can adjust these parameters (i.e. for big patient size), there are not standard clinical procedures that describe the process and this may affect the scanner adaptability. Furthermore, the noise is only one of the parameters that describe image quality in diagnostic imaging, and in future studies, it will be important to investigate the relationship between other image quality indexes (e.g., resolution, contrast) with radiation dose across patient populations.

\section{CONCLUSION}

We have shown that it is possible to find a theoretical 3D relationship between image noise, dose $\left(\mathrm{CTDI}_{\mathrm{vol}}\right)$, and patient size (effective diameter) in CT examinations. The comparison of real data with this function, in terms of RMSE in a 3D fit, is a metric that describes how different scanners are able to reproduce, for the same patient size, the same performance in terms of an important image quality parameter and in terms of CTDI ${ }_{\mathrm{vol}}$. These types of analyses are only possible through simultaneous image quality and dose monitoring, which can speak to the actual (as opposed to presumed) output of imaging systems. 


\section{REFERENCES}

[1] ICRP, 2007d "The 2007 Recommendations of the International Commission on Radiological Protection", ICRP Publication 103, Ann. ICRP 37, 2-4 (2007).

[2] ICRP, 2007 "Radiological Protection in Medicine" ICRP Publication 105, Ann. ICRP 37, 6 (2007).

[3] M. Gies, W.A. Kalender, H. Wolf, C. Suess, "Dose reduction in CT by anatomically adapted tube current modulation. Part I. Simulation studies", Medical Physics 26, 2235-2247 (1999).

[4] W.A. Kalender, H. Wolf, C. Suess, "Dose reduction in CT by anatomically adapted tube current modulation. Part II. Phantom measurements", Medical Physics 26, 2248-2253 (1999).

[5] M.K. Kalra, M.M. Maher, T.L. Toth, B. Schmidt, B.L. Westerman, H.T. Morgan, and S. Saini, "Techniques and applications of automatic tube current modulation for CT", Radiology 233, 649-657 (2004).

[6] T.Y. Lee, R.K. Chhem, "Impact of new technologies on dose reduction in CT", European Journal of Radiology 76, 28-35 (2010).

[7] D.B. Larson, L.L. Wang, D.J. Podberesky, M.J. Goske, "System for verifiable CT radiation dose optimization based on image quality. Part I. Optimization model", Radiology 269 167-176 (2013).

[8] O. Christianson, X. Li, D. P. Frush, E. Samei, "Automated size-specific CT dose monitoring program: Assessing variability in CT dose", Medical Physics 39, 7131-7138 (2012).

[9] O. Christianson, J. Winslow, D. P. Frush, E. Samei, "Automated Technique to Measure Noise in Clinical CT Examinations", AJR 205, W93-W99, 2015.

[10]X. Li, E. Samei, "Comparison of patient size-based methods for estimating quantum noise in CT images of the lung", Medical Physics 36, 541-546 (2009).

[11]N. Keat, "Report 05016 CT scanner automatic exposure control systems" ImPACT - London.

[12]"CCurve Fitting Toolbox ${ }^{\mathrm{TM}}$ User's guide”, The MathWorks Inc. Natick, (MA), USA, 2015.

[13]J.J. Sunderland and P.E. Christian, "Quantitative PET/CT scanner performance characterization based upon the Society on Nuclear Medicine and Molecular Imaging Clinical Trials Network Oncology Clinical Simulator Phantom", The Journal of Nuclear Medicine 56, 154-152 (2015). 